\title{
ARAÑAS ASOCIADAS A LA VEGETACIÓN ACUÁTICA DE DOS CIÉNAGAS DEL COMPLEJO CENAGOSO DEL BAJO SINÚ, CÓRDOBA-COLOMBIA
}

\section{SPIDERS ASSOCIATED TO AQUATIC VEGETATION IN TWO SWAMPS OF THE COMPLEX SWAMPS OF THE BAJO SINÚ, CÓRDOBA-COLOMBIA}

SALLEG P. GUSTAVO, ${ }^{1 *}$ Biol, QUIRÓS R. JORGE, ${ }^{1}$ M.Sc, WILD T. INDIRA ${ }^{1}$, Biol.

\section{Key words:}

Swamp, hidrological regime, spiders, structure, composition

\section{Palabras Clave:}

Ciénaga, régimen hidrológico, arañas, estructura, composición.
INFORMACIÓN Recibido: $19-06-2015$; Aceptado: 21-01-2016. Correspondencia autor: gsallegbio@gmail.com

\begin{abstract}
This research evaluated the structure and composition of spiders communities associated to aquatic vascular plants and their variation in the climate periods in two Complex Swamps of the Bajo Sinú. The spiders were collected monthly between November 2007 and June 2008; the capture methods used were manual collection and over PVC $(50 \times 50 \mathrm{~cm})$ quadrant; there were collected 943 individuals represented in 14 families and 55 morphospecies. From the spider families found, the most abundant were Araneidae, Lycosidae, and Salticidae; in terms of morphospecies richness Salticidae, Lycosidae, Theridiidae, Tetragnathidae and Linyphiidae were the best represented. To wetlands study, Momil swamp presented the mayority of morphospecies values, specific richness and diversity in relation to Purisima swamp; similar results had been obtained from transition period (rainy-dry) compared to dry and rainy periods. The results indicate that the spider communities associated to swampy ecosystems, are influenced by factors such complex and heterogeneity plants assembly, the hidrological regime and the level of ecosystem intervention.
\end{abstract}

\section{Resumen}

Se evaluó la estructura y composición de la comunidad de arañas asociada a plantas vasculares acuáticas y su variación en los periodos climáticos, en dos ciénagas del Complejo Cenagoso del Bajo Sinú. Los muestreos se realizaron entre los meses de noviembre de 2007 y julio de 2008, usando métodos de captura como colecta manual y sobre cuadrante de PVC $(50 \times 50 \mathrm{~cm})$. Se recolectaron en total 943 individuos, representados en 14 familias y 55 morfoespecies. Las familias más abundantes encontradas fueron Araneidae, Lycosidae y Salticidae. En cuanto a la riqueza de morfoespecies, Salticidae, Lycosidae, Theridiidae, Tetragnathidae y Linyphiidae fueron las mejor representadas. Para los humedales de estudio, la ciénaga de Momil presentó mayores valores de abundancia de morfoespecies, riqueza específica y diversidad, con respecto a la de Purísima. Resultados similares se obtuvieron para el periodo de transición (lluvias-sequía), comparado con la época seca y lluviosa. Los resultados indican que la comunidad de arañas asociada a ecosistemas cenagosos se ve influenciada por factores como la complejidad y heterogeneidad del ensamblaje de las plantas, el régimen hidrológico y el grado de intervención del ecosistema. 


\section{Introducción}

Los humedales son ecosistemas complejos que poseen características físicas, químicas y biológicas asociadas a los regímenes hídricos, ya sea de forma temporal o permanente (ROJAS et al., 2003). Son considerados sistemas de alta productividad vegetal, que disponen de agua temporalmente o totalmente durante todo el año, favoreciendo el desarrollo de una alta diversidad de fauna, flora y microorganismos (CASTELLANOS, 2006). Dentro de los humedales, las ciénagas son cuerpos de agua localizados en depresiones poco profundas y conectadas a los ríos mediante uno o más caños, de los cuales depende la renovación de sus aguas y el intercambio de materiales y organismos (ROLDÁN y RAMÍREZ, 2008). En las últimas décadas, estos sistemas han sufrido alteraciones por la actividad humana, lo que ha generado un impacto negativo sobre las formaciones vegetales y la fauna asociada (CVS, 2008), siendo de gran importancia generar información que sirva de apoyo en planes de manejo y monitoreo de este tipo de ecosistemas; de gran valor para la diversidad biológica local y regional.

Las arañas se caracterizan por ser un grupo megadiverso, en donde la estructura de sus comunidades demuestra relaciones especificas con las variaciones ambientales (TOTI et al., 2000). Su diversidad, densidad, distribución y composición están fuertemente relacionadas con aspectos como el tipo de vegetación, porcentaje de cobertura, rasgos espaciales y arquitectónicos los cuales definen la complejidad estructural de un hábitat (BALFOUR y RYPSTRA, 1998). Por tal razón, las arañas son sugeridas para el estudio de la biodiversidad en general y para la evaluación de hábitat naturales, siendo aceptadas en los estudios biológicos como indicadoras de calidad ambiental (JOCQUÉ y DIPPENAAR-SCHOEMAN, 2007).

Los estudios sobre arañas asociadas a ecosistemas dulceacuícolas son escasos, en el contexto internacional se destacan las investigaciones realizadas por LLINAS y JIMÉNEZ (2004) para arañas de humedales del sur de Baja California, México; y RAIZER y AMARAL (2001), quienes establecen la relación entre la complejidad estructural de macrófitas acuáticas y la diversidad de arañas asociadas, en el Pantanal de Matto Grosso, Brasil. Para Colombia, las menciones de arañas en humedales se reducen a resultados de investigaciones sobre la artropofauna asociada a comunidades vegetales en humedales altoandinos: DAPHNIA (1995), SDA y IDEA (2005), SÁNCHEZ y AMAT (2005), PONTIFICIA UNIVERSIDAD JAVERIANA y ACUEDUCTO DE BOGOTÁ (2009), ACUEDUCTO DE BOGOTÁ y CONSERVACIÓN INTENRACIONAL (2010), CLAVIJO y AMARILLO (2013). Para esta misma región CASTAÑEDA y MELO (2008) elaboran una guía de identificación a nivel de familia para las arañas presentes en el humedal La Conejera en Bogotá.

En Colombia los estudios sistemáticos de arañas asociadas a humedales son prácticamente nulos. La presente investigación tiene como objetivo conocer la estructura y composición de arañas asociadas a la vegetación acuática en dos sectores del Complejo Cenagoso del Bajo Sinú, y evaluar su relación con los períodos climáticos.

\section{Materiales y métodos.}

Área de estudio: El Complejo Cenagoso del Bajo Sinú (CCBS) se ubica sobre la margen derecha del Río Sinú, al norte del departamento de Córdoba, en las subregiones Bajo y Medio Sinú, entre los municipios de Lorica, Purísima, Momil, Cotorra, Chima y Ciénaga de Oro (Fig. 1). Posee un área de inundación de aproximadamente $440 \mathrm{~km}^{2}$. El clima de la zona se define como semiseco con una humedad relativa superior al $80 \%$ (SALAZAR, 2008). Presenta una precipitación promedio que varía entre 1198-1550 mm anuales; el periodo de lluvias se presenta de abril a noviembre, con las mayores precipitaciones entre agosto-septiembre, y el periodo seco va de diciembre a marzo, siendo febrero el mes con la menor precipitación (Fig. 2).

El estudio se realizó en dos sectores del CCBS, correspondientes a las ciénagas de Momil y Purísima.

Ciénaga de Momil: Se ubica en las coordenadas 9०13'34.96" N y $75^{\circ} 40^{\prime} 0.41^{\prime \prime} \mathrm{O}$, a una altitud de $17 \mathrm{~m}$ s.n.m (Fig.1). La llanura de inundación comprende un área de $27 \mathrm{~km}^{2}$, alcanzando profundidades de hasta $3 \mathrm{~m}$ (SALAZAR, 2008). La zona de estudio se caracteriza por conservar el espejo de agua, a pesar

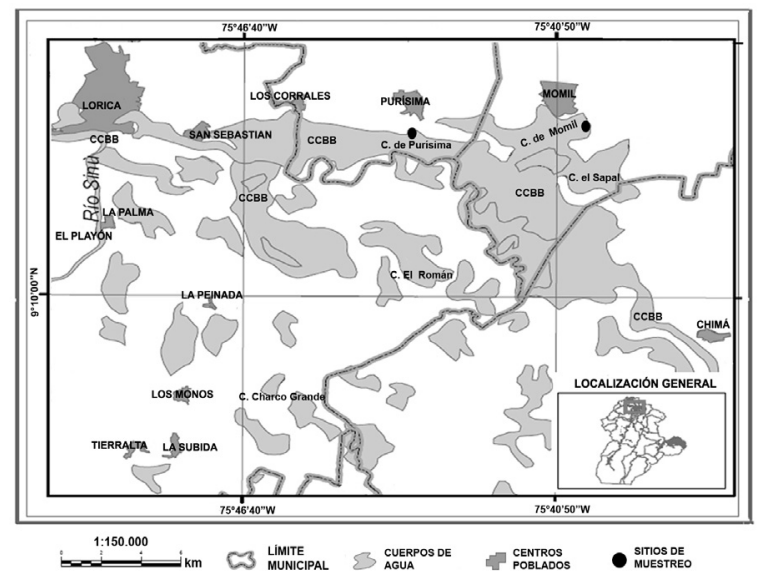

Figura 1. Área de estudio y ubicación de los sitios de muestreo en el CCBS. 


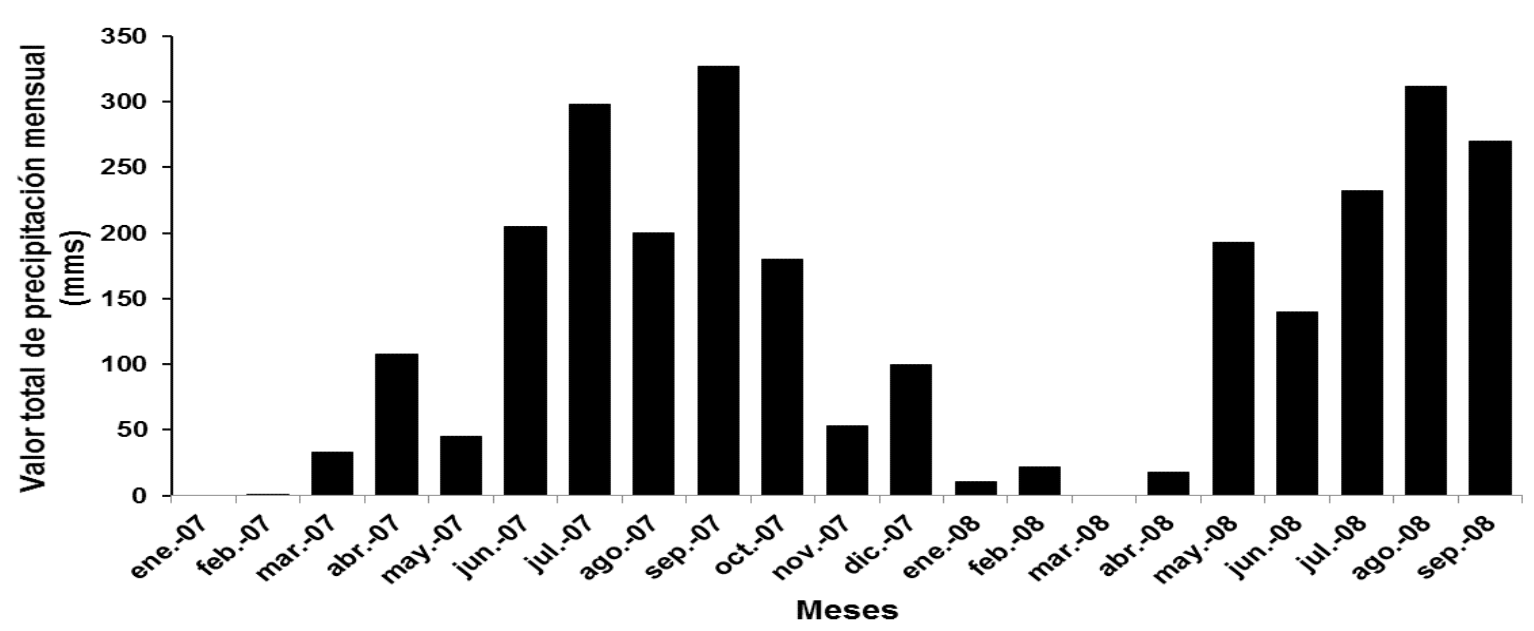

Figura 2. Variación pluviométrica en el CCBS durante el ciclo hidrológico anual (2007-2008). Fuente: IDEAM

de la disminución de los niveles de profundidad durante la época seca, razón por la cual se pueden encontrar bancos de macrófitas. Durante este tiempo se observó actividad agrícola, siendo predominante el cultivo de patilla.

La composición florística durante la época seca está representada por Eichhornia crassipes, Eichhornia azurea, Thalia geniculata, Ludwigia peploides, Heliotropium indicum, Euphorbia hypericifolia, Euphorbia sp., Mimosa pigra, Cyperus rotundus, Echinochloa colona y Cleome spinosa. Mientras que para la época de lluvias predominan Eichhornia crassipes, Salvinia auriculata, Pistia stratiotes, Ipomoea acuatica, Lersia hexandra. Neptunia oleracea, Eichhornia azurea, Cyperus odoratus, Thalia geniculata, Ludwigia helminthorrhiza, Hymenachne amplexicaulis, Poligonum hidropiperioides, Ceratophyllum echinatum, Najas arguta (MARTÍNEZ y ESPITIA, 2009).

Ciénaga de Purísima: Se encuentra ubicada a los $09^{\circ} 13^{\prime 28} .33^{\prime \prime} \mathrm{N}$ y $75^{\circ} 43^{\prime} 1.52$ " O, a una altitud de $20 \mathrm{~m}$ s.n.m (Fig. 1). Comprende una extensión de 14 km², con profundidades que oscilan entre los 0.5 y $5 \mathrm{~m}$ dependiendo de la época climática (PDM PURÍSIMA, 2012-2015). Es característico que durante los periodos de sequía el espejo de agua desaparezca en gran parte de su extensión; en la zona se observaron cultivos de patilla y actividad ganadera.

La composición florística para la época seca está representada por Solanum hirtum, Euphorbia sp., Euphorbia hypericifolia, Heliotropium indicum, Cleome spinosa, Mimosa pigra. Para el periodo de lluvias la composición es semejante a la ciénaga de Momil, sin embargo se hacen dominantes grandes parches monoespecíficos de E. crassipes (MARTíNEZ y ESPITIA, 2009).
Fase de Campo: Los muestreos se realizaron durante los meses de noviembre-diciembre de 2007 y febrerojulio de 2008. Durante este tiempo se realizó una salida mensual, de seis horas, para cada ciénaga de estudio. Para la recolección de las arañas, se instaló un transecto orientado de forma perpendicular a la orilla de la ciénaga, que se extendió desde la zona más somera del litoral hasta la zona comprendida por el espejo de agua. A lo largo del transecto se definieron estaciones de muestreo, distribuidas de la siguiente manera:

\section{Estación 1: Parte superior del litoral.}

Estación 2: Borde la ciénaga, interface tierra-agua.

Estación 3: Comprendió la zona del espejo de agua poco profunda.

Estación 4: Zona profunda.

En cada ciénaga, los muestreos fueron realizados por dos colectores, de manera simultánea, mediante los siguientes métodos:

Colecta sobre cuadrante: Consistió en capturar, de manera directa, las arañas utilizando un cuadrante de PVC de $50 \times 50 \mathrm{~cm}$, el cual fue adaptado con paredes laterales de acetato de $15 \mathrm{~cm}$ de alto para evitar que las arañas escaparan. Para la zona profunda, el cuadrante se adaptó con una malla (ojo de malla $120 \mu \mathrm{m}$ ), con el objetivo de extraer las plantas flotantes y posteriormente capturar las arañas (Adaptado de RAIZER y AMARAL, 2001). En total, se hicieron 10 réplicas del cuadrante a lo largo del transecto, distribuidas de la siguiente manera: las estaciones 1, 2 y 3 dos replicas cada una, y la estación 4 , cuatro réplicas, teniendo en cuenta que la profundidad dificultó la captura de las arañas.

Colecta manual: Consistió en la búsqueda minuciosa de arañas sobre la vegetación asociada al humedal, durante un tiempo efectivo de 15 minutos por colector 
en cada estación. Las arañas se depositaron en frascos plásticos de boca ancha, con alcohol al $70 \%$ para su conservación, y fueron etiquetados con un código asignado.

Fase de laboratorio: La determinación taxonómica se realizó en el Laboratorio de Entomología de la Universidad de Córdoba. Las arañas fueron separadas en familias usando claves taxonómicas de JOCQUÉ y DIPPENAAR- SCHOEMAN (2007), y posteriormente se identificaron morfoespecies, basándose en la observación de la genitalia y patrones de coloración. Para la determinación de géneros, en los casos que fue posible, se utilizaron las claves de BRESCOVIT (1997), HAZZI (2011), LEVI (1988) y FLÓREZ (s.f). Además se contó con la asesoría de Dimitar Dimitrov, investigador del Museo de Historia Natural de la Universidad de Oslo, en la identificación de géneros de Tetragnathidae. Los especímenes se depositaron en la sala de Colecciones Zoológicas de la Universidad de CórdobaCZUC.

Análisis de datos: El análisis de diversidad, entre ciénagas, entre periodos climáticos y entre estaciones de estudio, se realizó empleando el índice de riqueza específica de Margalef (Mg'), y los índices de estructura de Simpson (D'), Shannon-Wiener (H') y Pielou (J'). Los datos se analizaron estadísticamente aplicando la prueba W de Mann-Whitney (Wilcoxon) para comparar las ciénagas; y el Test de Kruskal-Wallis para hacer comparaciones entre los periodos climáticos y entre estaciones. Tanto el análisis de diversidad como el estadístico, se realizaron utilizando el programa PAST 3.06 (HAMMER, 2001). En relación a los periodos climáticos, los meses de noviembre y diciembre se consideraron de transición entre el periodo lluvias y sequía, debido a que en ellos se presentan aguas altas y se mantienen las coberturas vegetales. La eficiencia del inventario fue evaluada empleando los estimadores de riqueza ACE, ICE, Chao-2, Jackknife-1 y Bootstrap, suavizando las curvas con 100 aleatorizaciones, mediante el programa STIMATES 9.1 (COLWELL, 2013).

\section{Resultados}

Se recolectaron 943 individuos, agrupados en 14 familias y 55 morfoespecies. 517 individuos fueron adultos, $61,5 \%$ hembras, mientras que 426 individuos fueron registrados como juveniles. Las familias con mayor valor de abundancia fueron Araneidae (533), Lycosidae (186) y Salticidae (82), constituyendo el $84,9 \%$ de la abundancia total. Entre las menos representadas estuvieron Sparassidae (1) y Theraphosidae (1). En cuanto a la riqueza, las familias representadas con mayor número de morfoespecies fueron Salticidae
(16), Lycosidae (7), Theridiidae (6), Tetragnathidae (5), y Linyphiidae (5), mientras que Araneidae, Sparassidae y Theraphosidae presentaron la menor riqueza con una

Tabla 1. Riqueza y abundancia de arañas encontradas en las ciénagas de Momil y Purísima.

\begin{tabular}{ccc}
\hline Familia & Abundancia & Riqueza \\
\hline Araneidae & 533 & 1 \\
Lycosidae & 186 & 7 \\
Salticidae & 82 & 16 \\
Tetragnathidae & 37 & 5 \\
Theridiidae & 36 & 6 \\
Pisauridae & 33 & 2 \\
Ctenidae & 11 & 2 \\
Anyphaenidae & 9 & 2 \\
Linyphiidae & 7 & 5 \\
Oxyopidae & 3 & 3 \\
Clubionidae & 2 & 2 \\
Thomisidae & 2 & 2 \\
Sparassidae & 1 & 1 \\
Theraphosidae & 1 & 1 \\
\hline Total & $\mathbf{9 4 3}$ & $\mathbf{5 5}$ \\
\hline
\end{tabular}

morfoespecie cada una (Tabla 1).

En general, las arañas tejedoras fueron las más abundantes con 613 individuos (65\%) en comparación con las arañas errantes que estuvieron representadas por 330 individuos; en cuanto a riqueza, para las arañas cazadoras se identificaron 38 morfoespecies, mientras que para las constructoras de redes 17 .

Reportamos los géneros Hibana (Anyphaenidae), Ancylometes (Ctenidae), Oxyopes (Oxyopidae), Leucauge, Tetragnatha (Tetragnathidae), y la especie Alpaida veniliae (Araneidae) para ecosistemas cenagosos del CCBS.

Comparando los sitios de estudio, para la ciénaga de Momil se recolectaron en total 592 individuos y 49 morfoespecies, en contraste, la ciénaga de Purísima contó con 351 individuos y 26 morfoespecies. El tratamiento estadístico de los datos indicó diferencias significativas entre ambos humedales ( $p$-valor= $0,00028)$. Por otro lado, para Momil se obtuvieron mayores valores de riqueza $\left(\mathrm{Mg}^{\prime}=7,71\right)$ y diversidad $\left(H^{\prime}=2,13\right)$, así como una comunidad estructuralmente más homogénea $\left(D^{\prime}=0,32 ; J^{\prime}=0,55\right)$ en comparación con Purísima $\left(\mathrm{Mg}^{\prime}=4,34 ; \mathrm{H}^{\prime}=1,13 ; \mathrm{D}^{\prime}=0,64\right.$ y $\left.\mathrm{J}^{\prime}=0,35\right)$ (Fig. 3).

Para las épocas climáticas, el periodo de transición (262 individuos y $40 \mathrm{mfsp}$ ) y el periodo lluvioso (497 individuos y $26 \mathrm{mfsp}$ ) presentaron mayor abundancia 
de individuos y número de morfoespecies respecto al periodo seco (184 individuos y $22 \mathrm{mfsp}$ ). El análisis de los datos reveló la existencia de diferencias estadísticamente significativas entre los periodos estudiados ( $p$-valor $=0,0003$ ), siendo el periodo de transición (lluvias-sequía) significativamente diferente del periodo lluvioso y del periodo seco ( $p$-valor=0,0009 y 0,0004 respectivamente). El análisis de los atributos ecológicos arrojó un mayor valor de riqueza específica y diversidad para el periodo de transición $\left(\mathrm{Mg}^{\prime}=7,10 ; \mathrm{H}^{\prime}=\right.$ $2,79)$ con relación a la época seca $\left(\mathrm{Mg}^{\prime}=4,16 ; H^{\prime}=1,72\right)$, mientras que la época de lluvias obtuvo menores valores $\left(\mathrm{Mg}^{\prime}=4,13 ; \mathrm{H}^{\prime}=0,89\right)$. Este mismo comportamiento, se obtuvo para los valores de uniformidad de Pielou (J') durante cada periodo, que contrastan con la dominancia (D'), cuyo valor más alto se dio para el periodo de lluvias, lo que indica que gran parte de la abundancia está representada por unas pocas morfoespecies que son dominantes (Fig. 3).

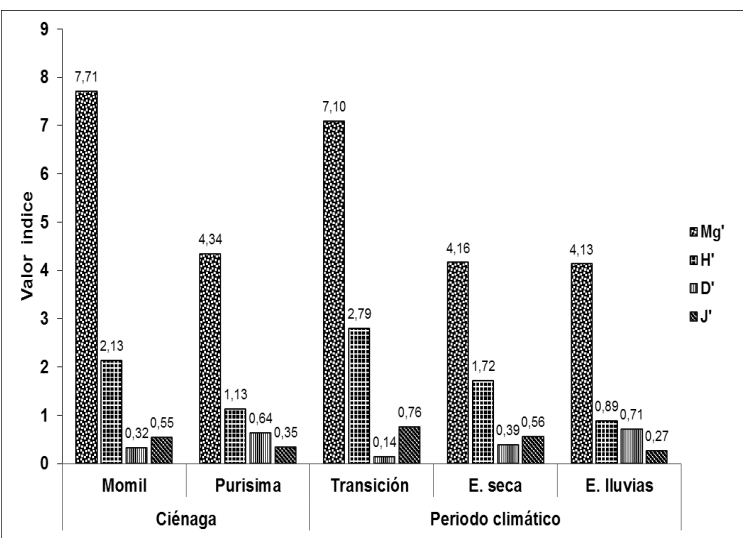

Figura 3. Índices de diversidad para las ciénagas de estudio y periodos climáticos.

Entre las estaciones de estudio, las de mayor abundancia de individuos fueron las estaciones 3 (206 individuos) y 4 (354 individuos) respecto a las estaciones 1 (185 individuos) y 2 (198 individuos); en cuanto al número de morfoespecies, la estaciones 2 (34 mfsp) y 4 (32 mfsp) estuvieron mayormente representadas en comparación con las estaciones 1 y 3 que contaron con 23 morfoespecies cada una. El análisis de los datos, no arrojó diferencias estadísticas significativas entre las estaciones de estudio ( $p$-valor= 0,15 ). En cuanto atributos ecológicos, los valores más altos para el índice de de riqueza específica y diversidad se presentaron en la estación $2\left(\mathrm{Mg}^{\prime}=6,34 ; \mathrm{H}^{\prime}=1,99\right)$; seguida de las estaciones $4\left(\mathrm{Mg}^{\prime}=5,44\right)$ y $1\left(\mathrm{Mg}^{\prime}=4,37\right)$ para el índice de Margalef, y las estaciones $3\left(H^{\prime}=1,99\right)$ y $4\left(H^{\prime}=1,56\right)$ para la diversidad de Shannon-Wiener. Por otro lado, las estaciones $2\left(D^{\prime}=0,35 ; J^{\prime}=0,57\right)$ y $3\left(D^{\prime}=0,4 ; J^{\prime}=\right.$ $0,56)$ mostraron mayor uniformidad para la forma como se distribuyen las abundancias entre las morfoespecies si se les compara con las estaciones $4\left(D^{\prime}=0,46\right.$; J'= $0,45)$ y $1\left(D^{\prime}=0,52 ; J^{\prime}=0,44\right)$, siendo esta última la que presentó valores más bajos de diversidad y uniformidad, y el valor más alto de dominancia (Fig. 4).

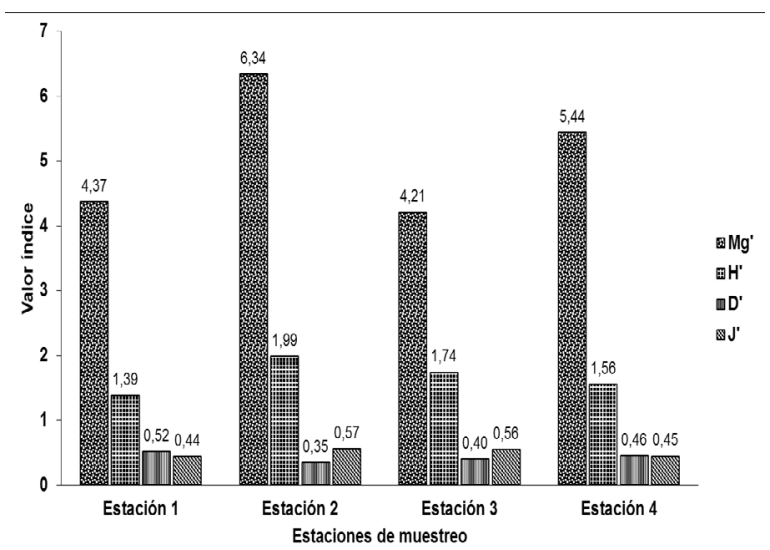

Figura 4. Índices de diversidad para las estaciones de muestreo.

Las morfoespecies con mayor número de individuos en esta investigación fueron Alpaida veniliae (533), Lycosidae sp1 (48), Pisauridae sp1 (30), Theridiidae sp5 (16), Tetragnatha sp1 (14), Tetragnatha sp2 (13), Salticidae sp2 (12), Salticidae sp7 (11), Lycosidae sp5 y Salticidae sp1 con diez individuos cada una, el resto de morfoespecies valores de abundancia menores a diez. En la Estación 1, las morfoespecies más abundantes fueron Alpaida veniliae (110), Lycosidae sp1, Pisauridae sp1, Tetragnatha sp1 y Lycosidae sp5 con cuatro individuo cada una; en la Estación 2, A. veniliae (106), Pisauridae sp1 (12) y Theridiidae sp5 (8); en la Estación 3, A. veniliae (117), Lycosidae sp1 (7), Theridiidae sp5 (7), Tetragnatha sp2 (6) y Salticidae sp1 (6); mientras que para la Estación 4, A. veniliae (200), Lycosidae sp1 (30), Pisauridae sp1(11), Tetragnatha sp1 (7) y Salticidae sp7 (6) fueron las más abundantes.

Los estimadores se calcularon combinando todos los métodos de colecta. Las curvas de acumulación de morfoespecies, para las ciénagas de estudio, no alcanzaron una asíntota, indicando que el número de morfoespecies aumentaría con un incremento en el número de unidades de muestreo. Según los estimadores empleados, la eficiencia de los muestreos, en promedio, fue de $70.4 \%$ para la ciénaga de Momil y de $75.2 \%$ para la ciénaga de Purísima. Para ambos sitios, la estimación más baja de morfoespecies se presentó con Bootstrap y ACE, y la más alta con Chao-2. Los singletons estuvieron representados por 19 (38.7\%) y 10 morfoespecies $(38.5 \%)$, y los doubletons por 6 $(12.2 \%)$ y 2 morfoespecies $(7.7 \%)$, para las ciénagas de Momil y Purísima respectivamente (Figs. 5 y 6). 


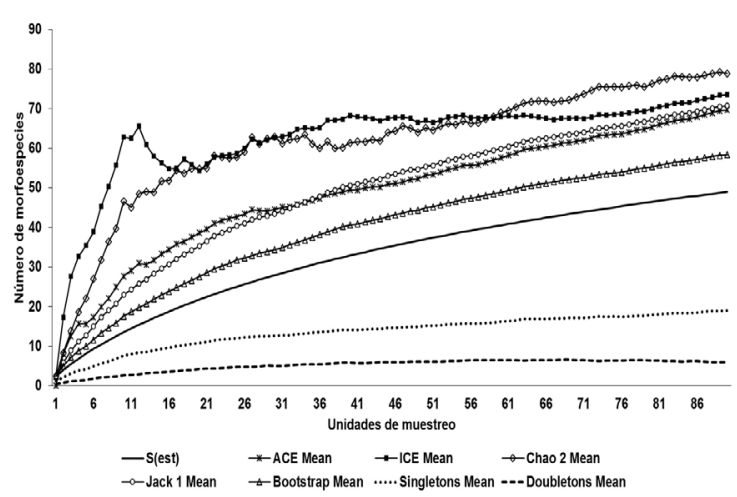

Figura 5 Curva de acumulación de morfoespecies de arañas para la ciénaga de Momil.

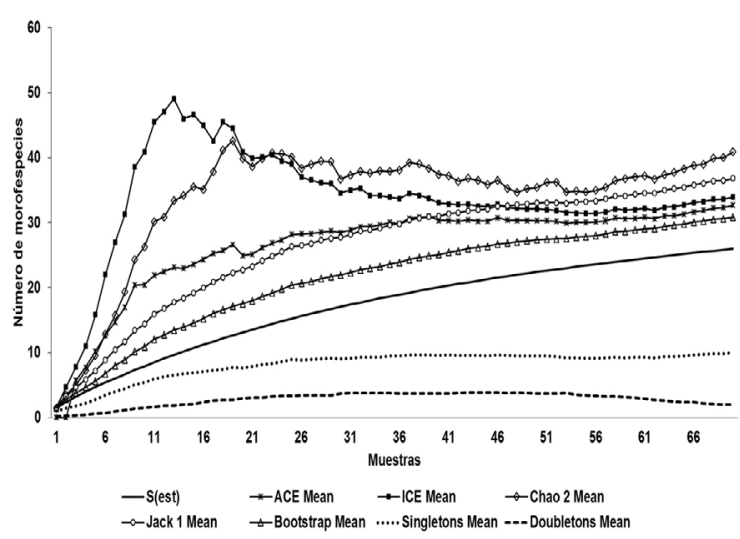

Figura 6. Curva de acumulación de morfoespecies de arañas para la ciénaga de Purísima.

\section{Discusión}

Este estudio estableció los primeros datos de diversidad de familias de arañas asociadas a la vegetación de ecosistemas cenagosos en el departamento de Córdoba. Desde el punto de vista taxonómico las familias de arañas encontradas en esta investigación representan el $40 \%$ del total de familias conocidas para el departamento (BEDOYA et al., 2011) y el $14 \%$ de las reportadas para Colombia (PERAFÁN et al., 2013).

Las familias con mayor número de individuos fueron Araneidae, Lycosidae y Salticidae; estas familias también han sido señaladas entre las más abundantes por DAPHNIA (1995), SDA y IDEA (2005), PONTIFICIA UNIVERSIDAD JAVERIANA y ACUEDUCTO DE BOGOTÁ (2009), ACUEDUCTO DE BOGOTÁ y CONSERVACIÓN INTERNACIONAL (2010), CLAVIJO y AMARILLO (2013) en humedales alto andinos de Bogotá. Así mismo, RAIZER y AMARAL (2001) reportan estas familias como las más abundantes en macrófitas del Pantanal de Matto Grosso en Brasil, y LLINAS y
JIMENEZ (2004) encuentran a las familias Thomisidae, Salticidae, Theridiidae, Anyphaenidae y Araneidae como las más dominantes de la región de humedales de Baja California, México.

Los humedales se caracterizan por presentar alta productividad y una elevada tasa de descomposición (ROLDÁN y RAMÍREZ, 2008), convirtiéndolos en lugares favorables para el establecimiento de cultivos, que en el caso de las ciénagas son plantados generalmente en época seca cuando disminuyen los niveles de agua (CVS, 2008). Los elevados valores de abundancia de Araneidae se deben a Alpaida veniliae; su dominancia en cultivos y la vegetación de la ciénaga le permitió tener una mayor presencia espaciotemporal, en ambas épocas climáticas, convirtiéndose en la especie dominante en los sitios de estudio. Se ha indicado que $A$. veniliae puede alcanzar altas densidades poblacionales y posee gran capacidad de depredación dentro de agroecosistemas (SAAVEDRA et al., 2007). Los anteriores aspectos permiten inferir que $A$. veniliae es la especie mejor adaptada a la dinámica de estos ecosistemas.

Los mayores valores de abundancia de la familia Lycosidae probablemente se deben al comportamiento errante de este grupo, que le permite vagar entre el ecosistema terrestre y acuático, siendo frecuente encontrarla en lugares de alta precipitación, humedad, o cercanas a cuerpos de agua (CHURCHILL, 1998). En cuanto a la familia Salticidae, además de su comportamiento errante, se caracteriza por tener una visión desarrollada y ser excelentes cazadoras diurnas, y fácilmente suelen migrar entre los diferentes estratos vegetales (FOELIX, 1996), lo que puede justificar la gran cantidad de individuos recolectados.

Entre las familias con mayor riqueza estuvieron Salticidae, Tetragnathidae, Theridiidae y Lycosidae, coincidiendo con lo observado por RAIZER y AMARAL (2001) en El Pantanal de Matto Grosso en Brasil, y LLINAS y JIMÉNEZ (2004) en Humedales de la Baja California en México, quienes también mencionan a estas familias entre las de mayor riqueza de especies. Las familias con mayor número morfoespecies e individuos, de este trabajo, también hacen parte del grupo de las más ricas y abundantes encontradas otros estudios importancia neotropical realizados en Colombia y Perú (RICO-G et al. 2005).

Esta investigación registra a las arañas tejedoras como las más abundantes, a diferencia de lo observado por RAIZER y AMARAL (2001) y LLINAS y JIMÉNEZ (2004), quienes reportan a las arañas errantes como las de mayor abundancia en humedales de Brasil y México respectivamente; la diferencia radica en elevado número de individuos presentado por $A$. veniliae en las ciénagas 
de estudio. Por otro lado, las arañas errantes fueron más diversas, coincidiendo con LLINAS y JIMÉNEZ (2004), quienes determinan que el éxito del gremio de las arañas errantes, en humedales, reside en aspectos como sus mínimos requerimientos en la arquitectura vegetal, su tolerancia ecológica que les permite transitar entre diferentes hábitats y su capacidad errante para salir en busca de sus presas; considerando ésta como una estrategia para prescindir de la competencia trófica con las arañas de redes.

La ciénaga de Momil exhibió mayores valores de diversidad, riqueza específica y un mayor número de arañas respecto a la de Purísima. MARTíNEZ y ESPITIA (2009) describen que la ciénaga de Momil presenta asociaciones vegetales más heterogéneas, a diferencia de Purísima donde la heterogeneidad del hábitat es mucho menor. Se ha determinado que la abundancia y diversidad de arañas varía dependiendo de la estructura y heterogeneidad del hábitat, indicando que a una mayor complejidad se presentan mayor número de individuos y riqueza de especies (BALFOUR y RYPSTRA, 1998). Además, la diversidad de arañas para Momil pudo ser estimulada por la presencia cercana de un relicto de bosque seco tropical (Cerro del Mohán), evidenciando así una mayor diversidad, teniendo en cuenta que las arañas presentan altas tasas de colonización de hábitat, debido a su capacidad de dispersión (HALAJ et al., 1998). Los anteriores aspectos concuerdan con SÁNCHEZ y AMAT (2005), quienes plantean que para los humedales en general, los valores de diversidad de artrópodos son condicionados por la acción conjunta de factores tales como: el régimen climático, tamaño y características del espejo de agua, heterogeneidad de los hábitats, la posición de los humedales entre ecosistemas terrestres y acuáticos, la disponibilidad de recursos alimenticios y el efecto de los depredadores.

Adicionalmente, las arañas son sensibles a los cambios en la comunidad de plantas que conforman su hábitat (BONTE et al., 2002) y son afectadas por el patrón y uso de la tierra (WEEKS y HOLTZER, 2000). En Purísima se observó un mayor grado de intervención antrópica producto de actividades agrícolas, la ganadería, y la construcción de jarillones; actividades que han degradado el ecosistema e inducido a la pérdida local de especies. Según CVS (2008), debido al avance y aumento de las actividades antrópicas en el Complejo Cenagoso del Bajo Sinú, las coberturas vegetales que existían se han reducido en un alto porcentaje, dando paso a paisajes con estructuras de beneficio para las sociedades que allí habitan, lo que ha conllevado a la pérdida progresiva de la fauna y la flora. McDONALD (2007) determina que alteraciones en la estructura y/o diversidad de plantas genera cambios en la diversidad y abundancia de artrópodos, y debido a que las arañas dependen enérgicamente de la disponibilidad de presas, la dinámica de sus comunidades suele sufrir alteraciones, afectando negativamente su estructura en el hábitat.

Asimismo, CHEN y TSO (2004) determinan que diferentes niveles de disturbios en la estructura del hábitat generan variaciones en la comunidad de arañas de un lugar a otro, por lo que una menor frecuencia de disturbios pueden proveer una mayor cantidad y diversidad de nichos disponibles para las arañas en hábitat complejos.

Por otro lado, los valores para el índice de ShannonWiener han sido clasificados de la siguiente manera: si el valor se encuentra por debajo de 1,5 , son considerados sitios con baja diversidad; si se encuentran entre $1,5 \mathrm{y}$ 3,5 , son sitios con diversidad media; y si superan los 3,5 , son sitios con alta diversidad (MAGURRAN, 2004). En Colombia, el intervalo dentro del que se encuentran los muestreos de arañas generalmente es el de diversidad media $(1,5-3,5)$ (SABOGAL, 2011). Por lo tanto, los valores de diversidad obtenidos para la ciénaga de Momil $(2,13)$ y Purísima $(1,13)$ permiten inferir que son sitios de diversidad media y baja, respectivamente.

En cuanto a los periodos climáticos, en la época de lluvia se encontraron más individuos, pero valores más bajos para los índices de Margalef y diversidad de Shannon-Wiener; siendo esto consecuencia de la baja equitatividad entre las morfoespecies, por la presencia $A$. veniliae en grandes proporciones $(71.4 \%)$ en comparación con la transición $(31.7 \%$ ) y el periodo seco $(51.6 \%)$. Teniendo en cuenta que en la transición entre la época lluviosa y seca (noviembre-diciembre) se presentaron los valores más altos de diversidad y uniformidad, infiere que a medida que transcurren los meses lluviosos existirá una tendencia al incremento de dichos valores, puesto que para las ciénagas estudiadas, durante los meses de aguas altas, se da un incremento en los valores de diversidad, cobertura y biomasa vegetal (MARTíNEZ y ESPÍTIA, 2009), condiciones que favorecen el establecimiento de las arañas.

En adición a lo anterior, LLINAS y JIMÉNEZ (2004) sostienen que las temporadas de lluvias generan condiciones de humedad que favorecen la presencia y proliferación de especies de arañas de exigencias mésicas, de otras de ambientes húmedos y principalmente de arañas ecológicamente tolerantes, que interactúan entre los cultivos y la vegetación natural. De igual manera el incremento de las lluvias estimula la floración y fructificación de la vegetación local que luego son invadidos por gran cantidad de invertebrados comensales, de los cuales muchos son presas de arañas. SÁNCHEZ y AMAT (2005) también demuestran que el gradiente espacio temporal dado por el régimen 
bimodal anual pluviométrico influye notablemente en las poblaciones de organismos, ya que algunas especies sincronizan su ritmo de fertilidad con dependencia de las épocas en que se presentan las fases hidrológicas. GRAVESEN (2000) determina que la intensidad de las inundaciones y las fluctuaciones en los niveles de agua en ecosistemas húmedos son factores importantes para la composición de especies de arañas.

En las ciénagas de estudio, se observó un mayor número de individuos y de especies vegetales en las estaciones ubicadas en el espejo de agua, excepto en la estación 4, donde suele encontrarse en gran abundancia Eichhornia crassipes; una mayor complejidad del hábitat, puede justificar los mayores valores de diversidad ShannonWiener para las estaciones 2 y 3 . Es preciso mencionar, que las arañas están más fuertemente relacionadas con la estructura de la vegetación presente en el litoral, que por la distancia a la que se encuentren del agua, siendo un factor más influyente en las arañas tejedoras (WILLOUGHBY y ANDERSON, 2004). Por otra parte, la no existencia de diferencias estadísticas significativas entre las estaciones de muestreo, pudo estar influenciada por su cercanía y a que la vegetación a lo largo del litoral suele conformar ensamblajes de macrófitas, permitiendo a las arañas desplazarse entre estaciones.

CODDINGTON et al. (2009) indican que en muestreos de artrópodos tropicales, el 30\% de todas las especies están representados por solamente un espécimen; estas especies, juegan un papel importante en la generación de la mayoría de los estimadores de riqueza, ya que están basados en su cuantificación (RICO-G et al., 2005). En esta investigación las curvas de acumulación no alcanzaron una estabilidad con el esfuerzo de muestreo empleado, en relación, el porcentaje de Singletons y doubletons, de ambas ciénagas, estuvo por encima del $30 \%$ respecto al total de individuos recolectados; conjuntamente el mayor número de especies raras, estuvo conformado por arañas errantes, siendo estas una fuente importante en el sesgo de los inventarios ya que no pueden ser consideradas como habitantes estrictos de un área específica (JIMÉNEZ y HORTAL, 2003).

Agradecimientos: Expresamos nuestros sinceros agradecimientos a: la Oficina de Administración de Investigación y Extensión de la Universidad de Córdoba y a Jorge Arias Ríos por haber permitido enmarcar esta investigación, en el capítulo de fauna asociada, del macroproyecto Estructura y Composición de Plantas Vasculares Acuáticas del departamento de Córdoba. A Dimitar Dimitrov por su valioso aporte en la identificación de géneros de la familia Tetragnathidae; A Roger Ayazo Berrocal, por su asesoría en la taxonomía del orden Araneae; A Juan Linares Arias, por sus orientaciones durante todo el desarrollo de la investigación; a Claudio Fernández Herrera, por facilitar los equipos necesarios y permitir que la revisión taxonómica se realizara en el Laboratorio de Entomología de la Universidad de Córdoba.

\section{Referencias}

ACUEDUCTO DE BOGOTÁ; CONSERVACIÓN INTERNACIONAL. 2010. Plan de Manejo Ambiental humedal Juan Amarillo. Versión final. Bogotá, Colombia.

BALFOUR, R.; RYPSTRA, A.1998. The influence of habitat structure on spider density in a no-till soybean agroecosystem. J. Arachnol 26:221-226.

BEDOYA, E.; SALLEG, G., QUIROS, J. 2011. Estado actual del conocimiento de arañas en (Arachnida:Araneae) en el departamento de Córdoba, Colombia. III Congreso Latinoamericano de Aracnología. Montenegro, Quindío, Colombia. Memorias y Resúmenes: 35-42.

BONTE, D.; LEON, B.; MAELFAIT, J. 2002. Spider assemblage structure and stability in a heterogeneous coastal dune system. J. Arachnol 30:331-343.

BRESCOVIT, A. 1997. Revisão de Anyphaeninae Bertkau a nivel de gêneros na região Neotropical (Araneae, Anyphaenidae). Revista brasileira de Zoologia 13 (1): 1-187.

CASTAÑEDA, J.; MELO, L. 2008. Guía para la observación y caracterización de arañas en el sendero del humedal La Conejera. Universidad Pedagógica Nacional. Bogotá, Colombia.

CASTELLANOS, C. 2006. Los ecosistemas de humedales en Colombia. Lunazul Universidad de Caldas. Disponible en: http://lunazul.ucaldas.edu.co/index2.php?option=com content\&task=view\&id=171\&. Consultado: 10-10-2009. 
CHEN, K.; TSO, I. 2004. Spider diversity on orchid island, Taiwan: a comparison between habitats receiving different degrees of human disturbance. Zoological Studies 43(3):598-611.

CLAVIJO, H.; AMARILLO, A. 2013. Variación taxonómica y funcional en la artropofauna asociada a comunidades vegetales en humedales altoandinos (Colombia). Revista Colombiana de Entomología 39(1):155-163.

COLWELL, R. 2013. EstimateS: Statistical estimation of species richness and shared species from samples. Version 9. User's Guide and application. Disponible en: http://purl.oclc.org/estimates. Consultado: 23-03-2015.

CODDINGTON, J; AGNARSSON, I; MILLER, J; KUNTNER, M; HORMIGA, G. 2009. Undersampling bias: the null hypothesis for singleton species in tropical arthropod surveys. Journal of Animal Ecology 78: 573-584.

CVS. 2008. PLAN de Manejo y Ordenamiento Ambiental del Complejo Cenagosos del Bajo Sinú. Medellín, Colombia.

CHURCHILL, T. 1998. Spiders as ecological indicators in the Australian tropics: Family distribution patterns along rainfall and grazing gradients. Proceedings of the 17th European colloquium of Arachnology, Edinburgh: 325-330.

DAPHNIA LTDA. 1995. Estudio ecológico y diseño del Plan de Manejo Ambiental del Humedal Juan Amarillo. Tomo I. Bogotá, Colombia.

FOELIX, R. 1996. Biology of spiders. Oxford University Press. New York, USA.

FLOREZ. s.f. Clave para géneros neotropicales de Oxypidae. (no publicado).

GRAVESEN, E. 2000. Spiders (Araneae) and other invertebrates groups as ecological indicators in Wetland Areas. Ekologia (Bratislava) 19(4):39-49.

HALAJ, J.; ROSS, D, MOLDENKE, A. 1998. Habitat structure and prey vailability as predictors of the abundance and community organization of piders in western Oregon forest canopies. J. Arachnol 26:203-220.

HAMMER, Ø; HARPER, D; RYAN, P. 2001. PAST: Paleontological statistics software package for education and data analysis. Palaeontologia Electronica 4(1).

HAZZI, N. 2011. Clave para los géneros de la familia Ctenidae de Suramérica (no publicado).

JIMÉNEZ; A.; HORTAL, J. 2003. Las curvas de acumulación de especies y la necesidad de evaluar la calidad de inventarios biológicos. Revista ibérica de aracnología 8:151-161.

JÓQUE, R.; DIPPENAAR-SCHOEMAN, A. 2007. Spider families of the world. Royal Museumfor Central Africa. Edition, ISBN. Tervuren, Bélgica.

LEVI, H. 1988. The neotropical orb-weaving spiders of the genus Alpaida (Araneae: Araneidae). Bulletin of the Museum Comparative Zoology 151: 365-487.

LLINAS, J.; JIMÉNEZ, M. 2004. Arañas de humedales del sur de Baja California, México. Anales del instituto de Biología, Universidad Autónoma de México, Serie Zoología 75(2):283-302.

MAGURRAN A. 2004. Measuring biological diversity. UK: Blackwell Science Ltd. 256 p.

McDONALD, B. 2007. Effects of vegetation structure on foliage dwelling spider assemblages in native and non-native oklahoma grassland hábitats. Proc. Okla. Acad. Sci. 87:85-88.

MARTINEZ, C; ESPITIA, V. 2009. Estructura y composición de las comunidades de plantas vasculares acuáticas de la Cienaga Grande de Lorica en los sectores de Momil y Purísima, Departamento de Córdoba. Trabajo de grado. Universidad de Córdoba. Montería, Colombia. 
PERAFÁN, C; SABOGAL, A; MORENO, J; GARCÍA, A; LUNA D; ROMERO, C; FLÓREZ, E. 2013. Diagnóstico del estado actual de la fauna de arácnidos y de su gestión en Colombia. Memorias del XL Congreso de la Sociedad Colombiana de Entomología. Bogotá: Sociedad Colombiana de Entomología: 308-335.

PLAN DE DESARROLLO MUNICIPAL DE PURISIMA 2012-2015. 2012. Municipio de Purísima de la Concepción, Córdoba. Córdoba.

PONTIFICIA UNIVERSIDAD JAVERIANA; ACUEDUCTO DE BOGOTA. 2009. Plan de Manejo Ambiental del humedal de Techo. Versión final. Bogotá, Colombia.

RAIZER, J.; AMARAL, M. 2001. Does The Structural Complexity Of Aquatic Macrophytes Explain The Diversity Of Associated Spider Assemblages?. J. Arachnol 29:227-237.

RICO-G, A.; BELTRÁN, J.; ÁLVAREZ, A.; FLÓREZ, E. 2005. Diversidad de arañas (ARACHNIDA: ARANEAE) en el Parque Nacional Natural Isla Gorgona, Pacífico Colombiano. Biota Neotropica 5(1):5-8.

ROJAS, M.; CAMPOS, M.; ALPIZAR, A.; BRAVO, J.; CÓRDOBA, R. 2003. El Cambio climático y los humedales en Centroamérica: implicaciones de la variación climática para los ecosistemas acuáticos y su manejo en la región. UICN. San José, Costa Rica.

ROLDÁN, G.; RAMÍREZ, J. 2008. Fundamentos de limnología Neotropical. Segunda edición. Editorial Universidad de Antioquia. Medellín, Colombia.

SAAVEDRA, E.; FLÓREZ, E.; FERNÁNDEZ, C. 2007. Capacidad de depredación y comportamiento de Alpaida veniliae (Araneae: Araneidae) en el cultivo de arroz. Revista Colombiana de Entomología 33(1):74-76.

SABOGAL, G. 2011. Estudio comparativo de las comunidades de arañas asociadas a bosques conservados y áreas intervenidas en el Santuario de flora y fauna Otún Quimbaya, Risaralda, Colombia. Trabajo de maestría. Universidad Nacional de Colombia. Bogotá.

SALAZAR, I. 2008. Lugar encantado de las aguas: Aspectos económicos de la Ciénaga Grande del Bajo Sinú. Centro de Estudios Económicos Regionales (CEER) del Banco de la República. Cartagena, Colombia.

SÁNCHEZ, D.; AMAT, G. 2005. Diversidad de la fauna de artrópodos terrestres en el humedal Jaboque, BogotáColombia. Caldasia 27(2):311-329.

SMA; IDEA. 2005. Plan de Manejo Ambiental del Parque Ecológico Distrital Humedal Tibanica. Versión final. Bogotá, Colombia.

TOTI, D. S.; COYLE, F. A.; MILLER, J. A. 2000. A structured inventory of Appalachian grass bald and heath bald spiders assemblages and a test of species richness estimator performance. J. Arachnol. 28:329-34.

WEEKS, R.; HOLTZER, T. 2000. Habitat and season in structuring ground-dwelling spiders (Araneae) Communities in a shrotgrass strppe ecosystem. Environ. Entomol 29(6):1164-1172.

WILLOUGHBY, R; ANDERSON, W. 2004. Interactions among land, water, and vegetation in shoreline arthropod communities. American journal of undergraduate research. 3(2):17-22. 